MPP-2008-59

\title{
The contribution of fermionic seesaws to the anomalous magnetic moment of leptons
}

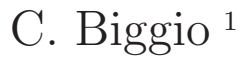 \\ Max-Planck-Institut für Physik, 80805 München, Germany
}

\begin{abstract}
We calculate the contribution to the anomalous magnetic moment of leptons in the type-I and type-III seesaw models. We show that, even if the scale of the new physics is pushed down to the electroweak scale, this contribution is not large enough to explain the measured discrepancy of the muon anomalous magnetic moment.
\end{abstract}

\footnotetext{
${ }^{1}$ biggio@mppmu.mpg.de
} 


\section{Introduction}

The anomalous magnetic moment of a spin-1/2 particle is defined as the difference between the gyromagnetic factor $g$ of that particle and its value in the Dirac theory. In formulae, $a=(g-2) / 2$, with $g$ defined by $\vec{\mu}=g \frac{e}{2 m} \vec{s}$, where $\vec{\mu}$ is the particle's magnetic moment, $\vec{s}$ its spin and $e$ and $m$ its electric charge and mass. Deviations from the Dirac's value $g=2$ are predicted in quantum theories due to loops effects.

The measurements of the electron and muon anomalous magnetic moments have recently reached an extraordinary precision, which has permitted, through the former, to give the most precise determination of the fine-structure constant $\alpha$ and, using the latter, to reveal a discrepancy between the standard model (SM) value and the experimental one. This discrepancy, that could be due to new physics beyond the SM, has motivated the calculation of $a_{\mu}$ in many different models like models with supersymmetry, extra dimensions, extended Higgs sector, additional gauge bosons... [1, 2]. Since new physics is required to explain neutrino masses, one may wonder if this could also affect observables like the anomalous magnetic moment. Even if it is usually believed that the new physics responsible for neutrino masses lies at a very high energy scale and therefore contributes in a negligible way to these low energy processes, it is not excluded that it can live at the energies which will be reached at near-future colliders like LHC. If this is the case, the contribution to low energy processes could be relevant and it is therefore interesting to study these effects.

The theoretical prediction of the electron anomalous magnetic moment is dominated by QED contributions which have been calculated up to four loops 1 [4. Adding to them the electroweak (EW) and the hadronic contributions [5] and comparing the result with the measured value [6]

$$
a_{e}^{e x p}=1159652180.85(76) \cdot 10^{-12}
$$

the most precise determination of $\alpha$ has been derived:

$$
\alpha^{-1}=137.035999709(96) \text {. }
$$

The present world average experimental value of the muon anomalous magnetic moment is given by [7]

$$
a_{\mu}^{e x p}=116592080(63) \cdot 10^{-11}
$$

On the theoretical side the reached precision is almost the same, where the error is dominated by the hadronic contribution. This can be extracted either from the hadronic $e^{+} e^{-}$annihilation data or from hadronic $\tau$ annihilation data. Depending on which of them is considered, the $a_{\mu}^{S M}$ ranges between $116591748(61) \cdot 10^{-11}$ [8] and

\footnotetext{
${ }^{1}$ See Ref. [3] for a short review on $e, \mu$ and $\tau$ anomalous magnetic moment and references therein.
} 
$116591961(70) \cdot 10^{-11}$ [9], which give rise to the following discrepancies 2:

$$
\begin{array}{ll}
\delta a_{\mu}=332(88) \cdot 10^{-11} & (3.8 \sigma) \\
\delta a_{\mu}=119(95) \cdot 10^{-11} & (1.3 \sigma) .
\end{array}
$$

Roughly, the difference between the SM prediction and the measured value is of $\mathcal{O}\left(10^{-9}\right)$. Any model of new physics able to contribute to the muon anomalous magnetic moment by a similar amount could be a good candidate to explain this discrepancy.

As for the tau anomalous magnetic moment, the experimental precision [11] is unfortunately too low compared with the theoretical one [3], so that no useful information can be obtained so far:

$$
\begin{gathered}
-0.052<a_{\tau}^{\text {exp }}<0.013 \\
a_{\tau}^{S M}=117721(5) \cdot 10^{-8} .
\end{gathered}
$$

In this paper we calculate the contribution to the anomalous magnetic moment of the leptons due to singlets (triplets) of fermions responsible for generating neutrino masses through the type-I (III) seesaw mechanism [12] ([13]). The organization of this paper is the following: in Sect. 2 we define our notations and calculate the anomalous magnetic moment due to triplets of fermions, in Sect. 3 we derive the result for the singlets case, while Sect. 4 contains our conclusions.

\section{The anomalous magnetic moment in the type-III seesaw model}

\subsection{The type-III seesaw Lagrangian}

The type-III seesaw model consists in the addition to the SM of SU(2) triplets of fermions with zero hypercharge, $\Sigma$. In this model at least two such triplets are necessary in order to have two non-vanishing neutrino masses, while there can be a contribution to the anomalous magnetic moment even with only one triplet. Therefore, in the following, we will not specify the number of triplets and our result will be independent of it. Following the notations of Ref. [14, the beyond-the-SM Lagrangian is given by

$$
\mathcal{L}=\operatorname{Tr}[\bar{\Sigma} i \not D \Sigma]-\frac{1}{2} \operatorname{Tr}\left[\bar{\Sigma} M_{\Sigma} \Sigma^{c}+\overline{\Sigma^{c}} M_{\Sigma}^{*} \Sigma\right]-\tilde{\phi}^{\dagger} \bar{\Sigma} \sqrt{2} Y_{\Sigma} L-\bar{L} \sqrt{2} Y_{\Sigma}^{\dagger} \Sigma \tilde{\phi},
$$

where $L \equiv(l, \nu)^{T}, \phi \equiv\left(\phi^{+}, \phi^{0}\right)^{T} \equiv\left(\phi^{+},(v+H+i \eta) / \sqrt{2}\right)^{T}$ with $v \equiv \sqrt{2}\left\langle\phi^{0}\right\rangle=246 \mathrm{GeV}$, $\tilde{\phi}=i \tau_{2} \phi^{*}, \Sigma^{c} \equiv C \bar{\Sigma}^{T}$ and where, for each fermionic triplet,

$$
\Sigma=\left(\begin{array}{cc}
\Sigma^{0} / \sqrt{2} & \Sigma^{+} \\
\Sigma^{-} & -\Sigma^{0} / \sqrt{2}
\end{array}\right) .
$$

\footnotetext{
${ }^{2}$ Intermediate values have been found in Refs. [10].
} 

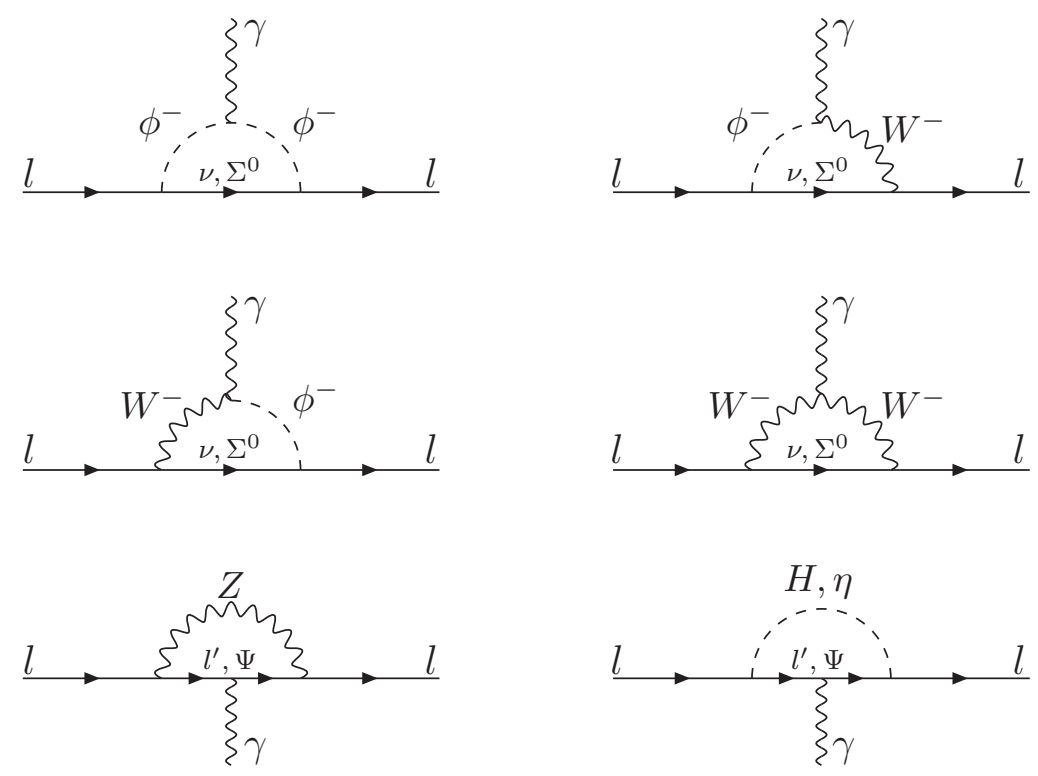

Figure 1: Diagrams contributing to the anomalous magnetic moment of the lepton $l$. $\phi^{ \pm}, \eta$ are the three Goldstone bosons associated with the $W^{ \pm}$and $Z$ bosons. $H$ stands for the physical Higgs boson.

Without loss of generality, in the following we will assume that we start from the basis where $M_{\Sigma}$ is real and diagonal. We express the four degrees of freedom of each charged triplet in terms of a single Dirac spinor $\Psi \equiv \Sigma_{R}^{+c}+\Sigma_{R}^{-}$, so that the field content of our model is given by the SM fields plus $n$ Majorana fermions $\Sigma^{0}$ (right-handed neutrinos) plus $n$ charged Dirac fermions $\Psi$. In the mass basis, the Lagrangian terms relevant to our calculation are the following

$$
\mathcal{L} \supset \mathcal{L}_{C C}+\mathcal{L}_{N C}+\mathcal{L}_{H, \eta}+\mathcal{L}_{\phi^{-}},
$$

where $\mathcal{L}_{C C}$ contains the charged current interactions among the charged leptons $l$, the charged fields $\Psi$, the neutrinos $\nu$ and the neutral fields $\Sigma^{0}, \mathcal{L}_{N C}$ are the neutral current interactions for charged fermions only and $\mathcal{L}_{H, \eta}$ and $\mathcal{L}_{\phi^{-}}$contain the interactions of the fermions with the Higgs and the Goldstone bosons (see Appendix A for details). In this model the neutrino mass matrix is given by:

$$
m_{\nu}=-\frac{v^{2}}{2} Y_{\Sigma}^{T} \frac{1}{M_{\Sigma}} Y_{\Sigma} .
$$

\subsection{Calculation of the anomalous magnetic moment}

The anomalous magnetic moment of a lepton $l$ is related to the photon-lepton vertex function $\Gamma^{\mu}$ as follows [15]:

$$
\begin{aligned}
\overline{u_{l}}(p-q) \Gamma^{\mu} u_{l}(p) & =\overline{u_{l}}(p-q)\left[\gamma^{\mu} F_{V}\left(q^{2}\right)+2 p^{\mu} F_{M}\left(q^{2}\right)+\ldots\right] u_{l}(p) \\
a_{l} & =-2 m_{l} F_{M}(0),
\end{aligned}
$$


where $p$ is the momentum of the incoming lepton and $q$ of the outcoming photon. We calculate the anomalous magnetic moment at $\mathcal{O}\left(\left(Y_{\Sigma} v / M_{\Sigma}\right)^{2}\right)$, which is a good approximation as long as $M_{\Sigma}$ is sufficiently big compared to $Y_{\Sigma} v$. The diagrams which contribute are depicted in Fig. 1. Grouping the amplitudes according to the fermion circulating in the loop, in the limit in which $m_{l}^{2} / M_{W}^{2} \rightarrow 0$, we have (see Appendix B for details):

$$
\begin{aligned}
T_{\nu}^{I I I} & =\frac{i e G_{F}^{S M}}{8 \sqrt{2} \pi^{2}} m_{l}\left\{\overline{u_{l}}(p-q)(2 p \cdot \varepsilon) u_{l}(p)\right\}\left[\frac{5}{3}+\frac{7}{6} \epsilon_{l l}-\frac{1}{2} \sum_{i} x_{\nu_{i}} V_{l i} V_{i l}^{\dagger}\right] \\
T_{l}^{I I I} & =\frac{i e G_{F}^{S M}}{8 \sqrt{2} \pi^{2}} m_{l}\left\{\overline{u_{l}}(p-q)(2 p \cdot \varepsilon) u_{l}(p)\right\} \times \\
& \times\left[\frac{2}{3}-4 \cos ^{2} \theta_{W}+\frac{8}{3} \cos ^{4} \theta_{W}+\epsilon_{l l}\left(-\frac{16}{3}+\frac{8}{3} \cos ^{2} \theta_{W}\right)\right] \\
T_{\Sigma}^{I I I} & =\frac{i e G_{F}^{S M}}{8 \sqrt{2} \pi^{2}} m_{l}\left\{\overline{u_{l}}(p-q)(2 p \cdot \varepsilon) u_{l}(p)\right\} \sum_{i} \frac{v^{2}}{2}\left(Y_{\Sigma}^{\dagger} M_{\Sigma}^{-1}\right)_{l i}\left(M_{\Sigma}^{-1} Y_{\Sigma}\right)_{i l} A\left(x_{i}\right)(16) \\
T_{\Psi}^{I I I} & =\frac{i e G_{F}^{S M}}{8 \sqrt{2} \pi^{2}} m_{l}\left\{\overline{u_{l}}(p-q)(2 p \cdot \varepsilon) u_{l}(p)\right\} \sum_{i} \frac{v^{2}}{2}\left(Y_{\Sigma}^{\dagger} M_{\Sigma}^{-1}\right)_{l i}\left(M_{\Sigma}^{-1} Y_{\Sigma}\right)_{i l} \times \\
\times & {\left[B\left(y_{i}\right)+C\left(z_{i}\right)\right] }
\end{aligned}
$$

where

$$
\begin{aligned}
A\left(x_{i}\right) & =\frac{-38+185 x_{i}-246 x_{i}^{2}+107 x_{i}^{3}-8 x_{i}^{4}+18\left(4-3 x_{i}\right) x_{i}^{2} \log x_{i}}{6\left(x_{i}-1\right)^{4}} \\
B\left(y_{i}\right) & =\frac{40-46 y_{i}-3 y_{i}^{2}+2 y_{i}^{3}+7 y_{i}^{4}+18\left(4-3 y_{i}\right) y_{i} \log y_{i}}{6\left(y_{i}-1\right)^{4}} \\
C\left(z_{i}\right) & =\frac{-16 z_{i}+45 z_{i}^{2}-36 z_{i}^{3}+7 z_{i}^{4}+6\left(3 z_{i}-2\right) z_{i} \log z_{i}}{6\left(z_{i}-1\right)^{4}}
\end{aligned}
$$

and $x_{\nu_{i}} \equiv \frac{m_{\nu_{i}}^{2}}{M_{W}^{2}}, x_{i} \equiv \frac{M_{\Sigma_{i}}^{2}}{M_{W}^{2}}, y_{i} \equiv \frac{M_{\Sigma_{i}}^{2}}{M_{Z}^{2}}, z_{i} \equiv \frac{M_{\Sigma_{i}}^{2}}{M_{H}^{2}}$. Moreover $V$ is the unitary PMNS leptonic mixing matrix and $\epsilon_{l l}=\sum_{i} \frac{v^{2}}{2}\left(Y_{\Sigma}^{\dagger}\right)_{l i} M_{\Sigma_{i}}^{-2}\left(Y_{\Sigma}\right)_{i l}$ is our expansion parameter 4 , not to be confused with $\varepsilon$ which represents the polarization of the photon. $\epsilon$ coincides with the coefficient of the unique dimension-six operator generated by the fermionic triplets once they have been integrated out and it is the unique combination of parameters which enters in all the low energy processes, a part from neutrino masses.

Notice that the $\epsilon$ and $x_{\nu_{i}}$-independent terms in Eq. (14)-(15) are precisely the 1loop EW contributions [16] and must be subtracted. The type-III seesaw contribution

\footnotetext{
${ }^{3} Y_{\Sigma}$ is $\mathcal{O}(1)$ or smaller. In particular, for the lowest allowed value of $M_{\Sigma}, 100 \mathrm{GeV}$ (see later), $Y_{\Sigma} \lesssim 10^{-2}$, to satisfy current EW bounds (see later).

${ }^{4}$ Notice that $x_{\nu_{i}} \sim \mathcal{O}(\epsilon)$, as it can be easily seen looking at Eq. (11).
} 
to the anomalous magnetic moment is then:

$$
\begin{aligned}
\Delta a_{l}^{I I I} & =\frac{G_{F}^{S M} m_{l}^{2}}{4 \sqrt{2} \pi^{2}}\left\{-\frac{1}{2} \sum_{i} x_{\nu_{i}} V_{l i} V_{i l}^{\dagger}+\sum_{i} \frac{v^{2}}{2}\left(Y_{\Sigma}^{\dagger} M_{\Sigma}^{-1}\right)_{l i}\left(M_{\Sigma}^{-1} Y_{\Sigma}\right)_{i l} \times\right. \\
& \left.\times\left[\frac{7}{6}-\frac{16}{3}+\frac{8}{3} \cos ^{2} \theta_{W}+A\left(x_{i}\right)+B\left(y_{i}\right)+C\left(z_{i}\right)\right]\right\} .
\end{aligned}
$$

The terms contained in the square parenthesis in the above expression constitute the loop factor which turns out to be a negative function which increases monotonically with $M_{\Sigma}$. Varying $M_{\Sigma}$ from $100.8 \mathrm{GeV}$ to infinity 5 , it varies between -2.86 (-3.18) to -1.11, taking $M_{H}=114.4 \mathrm{GeV}(250 \mathrm{GeV})$. Since $\sum_{i}\left(Y_{\Sigma}^{\dagger} M_{\Sigma}^{-1}\right)_{l i}\left(M_{\Sigma}^{-1} Y_{\Sigma}\right)_{i l}$ is a positive quantity, as well as $\sum_{i} x_{\nu_{i}} V_{l i} V_{i l}^{\dagger}$, this implies that the type-III seesaw contribution to the anomalous magnetic moment is always negative. This already tells us that, even if we lower the scale of the new physics, within this model we will not be able to explain the measured discrepancy in the muon sector.

Omitting flavour indices, from the seesaw formula, Eq. (11), one gets, in general, $\epsilon \sim m_{\nu} / M_{\Sigma} \sim 10^{-26}\left(10^{15} \mathrm{GeV} / M_{\Sigma}\right)$, where $m_{\nu} \sim \sqrt{\delta m_{\text {atm }}^{2}}$ has been used. On the other hand, since $x_{\nu} \sim \delta m_{\text {atm. }}^{2} / M_{W}^{2} \sim 10^{-24}$, for large $M_{\Sigma}$ the first term in Eq. (21) dominates and the contribution to the anomalous magnetic moment of leptons is negligible. When this relationship between $\epsilon$ and neutrino masses exists, even if $M_{\Sigma}$ is lowered to the electroweak scale gaining thus several orders of magnitudes, the type-III contribution will remain negligible. However there are cases where $\epsilon$ can be decoupled from neutrino masses or, in other words, where the smallness of these is obtained through cancellations which do not affect $\epsilon$ [18, 19]. In these cases the second term in Eq. (21) is enhanced and the first one can be neglected. Since we want to find an upper bound on the size of the total type-III contribution, we consider the case in which the term proportional to $x_{\nu_{i}}$ can be neglected and $M_{\Sigma}$ is low. In this case, and taking for the Higgs mass the lower limit $M_{H}=114.4 \mathrm{GeV}$, we have:

$$
\Delta a_{l}^{I I I}=\frac{G_{F}^{S M} m_{l}^{2}}{4 \sqrt{2} \pi^{2}}(-2.86) \epsilon_{l l} .
$$

What is now important in order to give an estimate of $\Delta a_{l}$ is the value of $\epsilon_{l l}$. We will discuss this in the next Section.

\subsection{The electron, muon and tau anomalous magnetic mo- ments}

The combination of the parameters of the type-III seesaw model which enters into this calculation, i. e. $\epsilon_{l l}$, is the same that appears in many EW processes like leptonic

\footnotetext{
${ }^{5}$ We choose, as lower limit on $M_{\Sigma}$, the LEP bound on the mass of new heavy charged particles [17], while the upper limit (infinity) is considered just in order to see which is the maximal value of the loop factor. In this case $\epsilon$ would go to zero, as well as $\Delta a_{l}^{I I I}$.
} 
and semileptonic decays of the $W$ and $Z$ bosons. Considering these processes, upper bounds on $\epsilon_{l l}$ have been derived in Ref. [18]: $\left|\epsilon_{e e}\right|<3 \cdot 10^{-3}$ and $\left|\epsilon_{\mu \mu}\right|=\left|\epsilon_{\tau \tau}\right|<4 \cdot 10^{-3}$. Taking them into account, we obtain the following constraints on $\Delta a_{l}^{I I I}$, in the case of "small" $M_{\Sigma}$ and with $M_{H}=114.4 \mathrm{GeV}(250 \mathrm{GeV})$ :

$$
\begin{aligned}
& \left|\Delta a_{e}^{I I I}\right|<4.66(5.18) \cdot 10^{-16} \\
& \left|\Delta a_{\mu}^{I I I}\right|<2.67(2.97) \cdot 10^{-11} \\
& \left|\Delta a_{\tau}^{I I I}\right|<7.55(8.39) \cdot 10^{-9} .
\end{aligned}
$$

We observe that the upper values obtained in Eqs. (23)-(25) are smaller than the theoretical errors in the SM computation, even if, for $\Delta a_{e}^{I I I}$ and $\Delta a_{\mu}^{I I I}$, they are of the same order of magnitude as the error in the EW part. Moreover, the value of $\left|\Delta a_{\mu}^{I I I}\right|$ obtained in this model is two orders of magnitude smaller than the measured discrepancy $\delta a_{\mu}$.

\section{The anomalous magnetic moment in the type-I seesaw model}

From the results obtained in the previous section it is now straightforward to derive the contribution to the anomalous magnetic moment in the type-I seesaw. Indeed in this case only singlet fermions are present, which can be identified with the fields $\Sigma^{0}$ of the type-III seesaw model. Consequently, among the diagrams depicted in Fig. 1, only the ones containing $\nu$ and $\Sigma^{0}$ as internal lines have to be considered.

The relevant couplings in this case are:

$$
\begin{array}{llll}
g_{L_{l \nu}}^{C C}=U_{0_{\nu \nu}} & g_{L_{l \Sigma}}^{C C}=Y_{\Sigma}^{\dagger} M_{\Sigma}^{-1} \frac{v}{\sqrt{2}} & g_{R_{l \nu}}^{C C}=0 & g_{R_{l \Sigma}}^{C C}=0 \\
g_{L_{\nu}}^{\phi^{-}}=m_{l} U_{0_{\nu \nu}} & g_{L_{\Sigma}}^{\phi^{-}}=m_{l} Y_{\Sigma}^{\dagger} M_{\Sigma}^{-1} \frac{v}{\sqrt{2}} & g_{R_{\nu}}^{\phi^{-}}=m_{\nu}^{*} U_{0_{\nu \nu}}^{*} & g_{R_{\Sigma}}^{\phi^{-}}=Y_{\Sigma}^{\dagger} \frac{v}{\sqrt{2}}\left(1-\frac{\epsilon^{\prime}}{2}\right)
\end{array} .
$$

Again, grouping the diagrams according to the fermion circulating in the loop and making the same approximations we did in the previous section, we obtain, at $\mathcal{O}(\epsilon)$ :

$$
\begin{aligned}
T_{\nu}^{I} & =\frac{i e G_{F}^{S M}}{8 \sqrt{2} \pi^{2}} m_{l}\left\{\overline{u_{l}}(p-q)(2 p \cdot \varepsilon) u_{l}(p)\right\}\left[\frac{5}{3}-\frac{5}{3} \epsilon_{l l}-\frac{1}{6} \sum_{i} x_{\nu_{i}} V_{l i} V_{i l}^{\dagger}\right] \\
T_{\Sigma}^{I} & =\frac{i e G_{F}^{S M}}{8 \sqrt{2} \pi^{2}} m_{l}\left\{\overline{u_{l}}(p-q)(2 p \cdot \varepsilon) u_{l}(p)\right\} \sum_{i} \frac{v^{2}}{2}\left(Y_{\Sigma}^{\dagger} M_{\Sigma}^{-1}\right)_{l i}\left(M_{\Sigma}^{-1} Y_{\Sigma}\right)_{i l} D\left(x_{i}\right)
\end{aligned}
$$

where

$$
D\left(x_{i}\right)=\frac{10-55 x_{i}+90 x_{i}^{2}-37 x_{i}^{3}-8 x_{i}^{4}+6\left(7 x_{i}-4\right) x_{i}^{2} \log x_{i}}{6\left(x_{i}-1\right)^{4}} .
$$

In Eq. (27) we recognize again the EW contribution of neutrinos which we have to subtract. The contribution to the anomalous magnetic moment of type-I seesaw is 
then:

$$
\Delta a_{l}^{I}=\frac{G_{F}^{S M} m_{l}^{2}}{4 \sqrt{2} \pi^{2}}\left\{\sum_{i} \frac{v^{2}}{2}\left(Y_{\Sigma}^{\dagger} M_{\Sigma}^{-1}\right)_{l i}\left(M_{\Sigma}^{-1} Y_{\Sigma}\right)_{i l}\left[D\left(x_{i}\right)-\frac{5}{3}\right]-\frac{1}{6} \sum_{i} x_{\nu_{i}} V_{l i} V_{i l}^{\dagger}\right\} .
$$

As before, we are interested in cases where the first term dominates over the second, so that, from now on, we will neglect the latter. The terms contained in the square parenthesis in the above expression constitute the loop factor which turns out to be a negative monotonically-decreasing function. Then, also in this model, the contribution to the anomalous magnetic moment of the leptons is always negative and the measured deviation in the muon sector can not thus be explained.

Also for the type-I seesaw, considering EW decays, it is possible to put bounds on $\epsilon_{l l}$. These, which are slightly larger than the ones obtained in the type-III case, $\left|\epsilon_{l l}\right|<10^{-2}$, have been derived in Ref. [20]. Taking them into account and considering the case in which $M_{\Sigma}=100 \mathrm{GeV}(1 \mathrm{TeV})$, we obtain, for each lepton:

$$
\begin{aligned}
& \left|\Delta a_{e}^{I}\right|<6.18 \cdot 10^{-16} \quad\left(1.54 \cdot 10^{-15}\right) \\
& \left|\Delta a_{\mu}^{I}\right|<2.65 \cdot 10^{-11} \quad\left(6.63 \cdot 10^{-11}\right) \\
& \left|\Delta a_{\tau}^{I}\right|<7.50 \cdot 10^{-9} \quad\left(1.87 \cdot 10^{-8}\right) .
\end{aligned}
$$

We observe that also in this model the new contributions are generically smaller than the SM error. However, if we consider $\left|\Delta a_{\tau}^{I}\right|$, for masses of order of $1 \mathrm{TeV}$ or higher, we see that it can be comparable with the present SM error.

\section{Conclusions}

Motivated by the deviation from the SM value of the measured anomalous magnetic moment of the muon and considering that new physics beyond the SM is required to explain neutrino masses, we have calculated the contribution to the anomalous magnetic moment of the leptons in two classes of models for generating neutrino masses, i. e. the type-I and type-III seesaw models.

We have found that, even if the scale of such new physics is just behind the corner, in the reach of future accelerators like LHC, its contribution to the anomalous magnetic moment of the leptons is generically smaller than the theoretical errors in the SM calculation, rendering thus impossible to distinguish from the SM. Notice that what forces this contribution to be so small are the bounds on $\epsilon_{l l}$ coming from other EW processes. Since in seesaw models like these the combination of the parameters of the new physics which enters into low energy processes is unique, the strong bounds coming from various EW processes apply, making impossible to use this new physics to explain the discrepancy of the muon anomalous magnetic moment. This is not the case for example in supersymmetric theories [2], where the much larger parameters 
space makes it possible that different combinations of them affect different processes, permitting thus to evade EW bounds.

We can thus conclude that, even if new physics responsible for neutrino mass generation like fermionic singlets or triplets will be discovered at low scale, its contribution to the anomalous magnetic moment of the leptons will anyway be extremely small.

\section{Note added}

During the completion of this work, Ref. [21] appeared, calculating the anomalous magnetic moment of the muon in three low-scale seesaw models. Our results differ from the ones presented in that paper in the case of type-III seesaw by one order of magnitude 6. Firstly, in Ref. [21] only the Higgs diagram is calculated, arguing that this is the relevant one. However, from our Eqs. (14)-(21) it can be seen that all the diagrams give a contribution of the same order of magnitude. This is a difference, but it does not lead to one order of magnitude of discrepancy. This discrepancy comes from the fact that in Ref. [21] existing bounds on $\epsilon_{l l}$ have not been taken into account. If we take the result of Ref. [21], Eqs. (20)-(21), re-express it in terms of $\epsilon$ and take the limit for small $M_{\Sigma}$, we obtain a formula similar to our Eq. (22), but with the numerical factor being even one order of magnitude smaller. So it seems to be impossible to get a contribution of the order of $10^{-10}$, as claimed in that paper, if the existing bounds are taken into account.

\section{Acknowledgments}

We acknowledge discussions with S. Béjar, E. Fernandéz-Martínez, T. Hambye, A. Ibarra, M. Passera, S. Rigolin and S. Vempati. We especially thank M. Papucci for useful discussions and for precious comments on the first version of this manuscript.

\footnotetext{
${ }^{6} \mathrm{~A}$ small difference is also present in the type-I case, due to an extra factor 2 in the diagram containing two Goldstone bosons circulating in the loop.
} 


\section{Appendix A}

The Lagrangian terms in Eq. (10), which are the ones relevant for our calculation, are:

$$
\begin{aligned}
& \mathcal{L}_{C C}=\frac{g}{\sqrt{2}}\left(\begin{array}{ll}
\bar{l} & \bar{\Psi}
\end{array}\right) \gamma^{\mu} W_{\mu}^{-}\left(P_{L} g_{L}^{C C}+P_{R} g_{R}^{C C} \sqrt{2}\right)\left(\begin{array}{c}
\nu \\
\Sigma^{0}
\end{array}\right)+\text { h.c. } \\
& \mathcal{L}_{N C}=\frac{g}{\cos \theta_{W}}\left(\begin{array}{ll}
\bar{l} & \bar{\Psi}
\end{array}\right) \gamma^{\mu} Z_{\mu}\left(P_{L} g_{L}^{N C}+P_{R} g_{R}^{N C}\right)\left(\begin{array}{c}
l \\
\Psi
\end{array}\right) \\
& \mathcal{L}_{H, \eta}=\frac{g}{2 M_{W}}\left(\begin{array}{ll}
\bar{l} & \bar{\Psi}
\end{array}\right) H\left(P_{L} g_{L}^{H}+P_{R} g_{R}^{H}\right)\left(\begin{array}{c}
l \\
\Psi
\end{array}\right) \\
& +i \frac{g}{2 M_{W}}\left(\begin{array}{ll}
\bar{l} & \bar{\Psi}
\end{array}\right) \eta\left(P_{L} g_{L}^{\eta}+P_{R} g_{R}^{\eta}\right)\left(\begin{array}{c}
l \\
\Psi
\end{array}\right) \\
& \mathcal{L}_{\phi^{-}}=-\phi^{-} \bar{l} \frac{g}{\sqrt{2} M_{W}}\left\{\left(P_{L} g_{L_{\nu}}^{\phi^{-}}+P_{R} g_{R_{\nu}}^{\phi^{-}}\right) \nu+\left(P_{L} g_{L_{\Sigma}}^{\phi^{-}}+P_{R} g_{R_{\Sigma}}^{\phi^{-}}\right) \Sigma^{0}\right\}+\text { h.c. }
\end{aligned}
$$

with

$$
\begin{aligned}
& g_{L}^{C C}=\left(\begin{array}{cc}
g_{L_{l \nu}^{C C}}^{C C} & g_{L_{l \Sigma}}^{C C} \\
g_{L_{\Psi \nu}}^{C C} & g_{L_{\Psi \Sigma}}^{C C}
\end{array}\right)=\left(\begin{array}{cc}
(1+\epsilon) U_{0_{\nu \nu}} & -Y_{\Sigma}^{\dagger} M_{\Sigma}^{-1} \frac{v}{\sqrt{2}} \\
0 & \ldots
\end{array}\right) \\
& g_{R}^{C C}=\left(\begin{array}{cc}
g_{R_{l \nu}^{C C}}^{C C} & g_{R_{l \Sigma}}^{C C} \\
g_{R_{\Psi \nu}}^{C C} & g_{R_{\Psi \Sigma}}^{C C}
\end{array}\right)=\left(\begin{array}{cc}
0 & -m_{l} Y_{\Sigma}^{\dagger} M_{\Sigma}^{-2} v \\
-M_{\Sigma}^{-1} Y_{\Sigma}^{*} U_{0_{\nu \nu}}^{*} \frac{v}{\sqrt{2}} & \cdots
\end{array}\right) \\
& g_{L}^{N C}=\left(\begin{array}{cc}
g_{L_{l l}}^{N C} & g_{L_{l \Psi}}^{N C} \\
g_{L_{\Psi l}}^{N C} & g_{L_{\Psi \Psi}}^{N C}
\end{array}\right)=\left(\begin{array}{cc}
\frac{1}{2}-\cos ^{2} \theta_{W}-\epsilon & \frac{1}{2} Y_{\Sigma}^{\dagger} M_{\Sigma}^{-1} v \\
\frac{1}{2} M_{\Sigma}^{-1} Y_{\Sigma} v & \ldots
\end{array}\right) \\
& g_{R}^{N C}=\left(\begin{array}{cc}
g_{R_{l l}}^{N C} & g_{R_{l}}^{N C} \\
g_{R_{\Psi l} C}^{N C} & g_{R_{\Psi}}^{N C}
\end{array}\right)=\left(\begin{array}{cc}
1-\cos ^{2} \theta_{W} & m_{l} Y_{\Sigma}^{\dagger} M_{\Sigma}^{-2} v \\
M_{\Sigma}^{-2} Y_{\Sigma} m_{l} v & \ldots
\end{array}\right) \\
& g_{L}^{H}=\left(\begin{array}{cc}
g_{L_{l l}}^{H} & g_{L_{l \Psi}}^{H} \\
g_{L_{\Psi l}}^{H} & g_{L_{\Psi \Psi}}^{H}
\end{array}\right)=\left(\begin{array}{cc}
m_{l}(3 \epsilon-1) & -m_{l} Y_{\Sigma}^{\dagger} M_{\Sigma}^{-1} v \\
-Y_{\Sigma} v(1-\epsilon)-M_{\Sigma}^{-2} Y_{\Sigma} m_{l}^{2} v & \ldots
\end{array}\right) \\
& g_{R}^{H}=\left(\begin{array}{cc}
g_{R_{l l}}^{H} & g_{R_{l \Psi}}^{H} \\
g_{R_{\Psi l}}^{H} & g_{R_{\Psi \Psi}^{H}}^{H}
\end{array}\right)=\left(\begin{array}{cc}
(3 \epsilon-1) m_{l} & -(1-\epsilon) Y_{\Sigma}^{\dagger} v-m_{l}^{2} Y_{\Sigma}^{\dagger} M_{\Sigma}^{-2} v \\
-M_{\Sigma}^{-1} Y_{\Sigma} m_{l} v & \ldots
\end{array}\right) \\
& g_{R}^{\eta}=\left(\begin{array}{cc}
g_{R_{l l}}^{\eta} & g_{R_{l \Psi}}^{\eta} \\
g_{R_{\Psi l}}^{\eta} & g_{R_{\Psi \Psi}}^{\eta}
\end{array}\right)=\left(\begin{array}{cc}
-(\epsilon+1) m_{l} & (1-\epsilon) Y_{\Sigma}^{\dagger} v-m_{l}^{2} Y_{\Sigma}^{\dagger} M_{\Sigma}^{-2} v \\
-M_{\Sigma}^{-1} Y_{\Sigma} m_{l} v & \ldots
\end{array}\right) \\
& g_{L}^{\eta}=\left(\begin{array}{cc}
g_{L_{l l}}^{\eta} & g_{L_{l \Psi}}^{\eta} \\
g_{L_{\Psi l}}^{\eta} & g_{L_{\Psi \Psi}}^{\eta}
\end{array}\right)=\left(\begin{array}{cc}
m_{l}(\epsilon+1) & m_{l} Y_{\Sigma}^{\dagger} M_{\Sigma}^{-1} v \\
-Y_{\Sigma} v(1-\epsilon)+M_{\Sigma}^{-2} Y_{\Sigma} m_{l}^{2} v & \ldots
\end{array}\right)
\end{aligned}
$$

and

$$
\left\{\begin{array} { l } 
{ g _ { L _ { \nu } } ^ { \phi ^ { - } } = m _ { l } U _ { 0 _ { \nu \nu } } } \\
{ g _ { R _ { \nu } } ^ { \phi ^ { - } } = - ( 1 - \epsilon ) m _ { \nu } ^ { * } U _ { 0 _ { \nu \nu } } ^ { * } }
\end{array} \quad \left\{\begin{array}{l}
g_{L_{\Sigma}}^{\phi^{-}}=m_{l} Y_{\Sigma}^{\dagger} M_{\Sigma}^{-1} \frac{v}{\sqrt{2}} \\
g_{R_{\Sigma}}^{\phi^{-}}=(1-\epsilon) Y_{\Sigma}^{\dagger} \frac{v}{\sqrt{2}}\left(1-\frac{\epsilon^{\prime *}}{2}\right)-\sqrt{2} m_{\nu}^{*} Y_{\Sigma}^{T} M_{\Sigma}^{-1} v
\end{array} .\right.\right.
$$

The dots in the previous equations refer to $\Sigma^{0}-\Sigma^{0}$ and $\Psi-\Psi$ interactions which do not contribute to the process we are considering. Moreover $\epsilon=\frac{v^{2}}{2} Y_{\Sigma}^{\dagger} M_{\Sigma}^{-2} Y_{\Sigma}, \epsilon^{\prime}=$ $\frac{v^{2}}{2} M_{\Sigma}^{-1} Y_{\Sigma} Y_{\Sigma}^{\dagger} M_{\Sigma}^{-1}$ and $U_{0 \nu \nu}=\left(1-\frac{\epsilon}{2}\right) V$, where $V$ is the lowest order neutrino mixing matrix (PMNS matrix) which is unitary; finally we take $\sin ^{2} \theta_{W}=0.23$. 


\section{Appendix B}

Performing the calculation in the 't Hooft-Feynman gauge, after loop integration, the various amplitudes, at $\mathcal{O}(\epsilon)$ and neglecting terms proportional to $x_{l}=m_{l}^{2} / M_{W}^{2}, y_{l}=$ $m_{l}^{2} / M_{Z}^{2}, z_{l}=m_{l}^{2} / M_{H}^{2}$ and $m_{l}^{2} / M_{\Sigma}^{2}$ that will give irrelevant contributions, are:

$$
\begin{aligned}
& T_{\nu_{i}}^{\phi^{-} / W^{-}}=\frac{i e G_{F}^{S M}}{8 \sqrt{2} \pi^{2}} m_{l}\left\{\overline{u_{l}}(p-q) P_{R}(2 p \cdot \varepsilon) u_{l}(p)\right\}\left[\left(1+\frac{\epsilon}{2}\right)_{l \alpha} V_{\alpha i} V_{i \beta}^{\dagger}\left(1-\frac{\epsilon}{2}\right)_{\beta l}\right] F_{1}\left(x_{\nu_{i}}\right) \\
& T_{\nu_{i}}^{W^{-} / \phi^{-}}=\frac{i e G_{F}^{S M}}{8 \sqrt{2} \pi^{2}} m_{l}\left\{\overline{u_{l}}(p-q) P_{L}(2 p \cdot \varepsilon) u_{l}(p)\right\}\left[\left(1-\frac{\epsilon}{2}\right)_{l \alpha} V_{\alpha i} V_{i \beta}^{\dagger}\left(1+\frac{\epsilon}{2}\right)_{\beta l}\right] F_{1}\left(x_{\nu_{i}}\right) \\
& T_{\nu_{i}}^{W^{-} / W^{-}}=\frac{i e G_{F}^{S M}}{8 \sqrt{2} \pi^{2}} m_{l}\left\{\overline{u_{l}}(p-q)(2 p \cdot \varepsilon) u_{l}(p)\right\}\left[\left(1+\frac{\epsilon}{2}\right)_{l \alpha} V_{\alpha i} V_{i \beta}^{\dagger}\left(1+\frac{\epsilon}{2}\right)_{\beta l}\right] F_{2}\left(x_{\nu_{i}}\right) \\
& T_{\nu_{i}}^{\phi^{-} / \phi^{-}}=\frac{i e G_{F}^{S M}}{8 \sqrt{2} \pi^{2}} m_{l}\left\{\overline{u_{l}}(p-q)(2 p \cdot \varepsilon) u_{l}(p)\right\}\left[\left(1-\frac{\epsilon}{2}\right)_{l \alpha} V_{\alpha i} V_{i \beta}^{\dagger}\left(1-\frac{\epsilon}{2}\right)_{\beta l}\right] x_{\nu_{i}} F_{3}\left(x_{\nu_{i}}\right) \\
& T_{\Sigma_{i}}^{\phi^{-} / W^{-}}=-\frac{i e G_{F}^{S M}}{8 \sqrt{2} \pi^{2}} m_{l}\left\{\overline{u_{l}}(p-q)\left(2 P_{L}+P_{R}\right)(2 p \cdot \varepsilon) u_{l}(p)\right\} \frac{v^{2}}{2}\left(Y_{\Sigma}^{\dagger} M_{\Sigma}^{-1}\right)_{l i}\left(M_{\Sigma}^{-1} Y_{\Sigma}\right)_{i l} F_{1}\left(x_{i}\right) \\
& T_{\Sigma_{i}}^{W^{-} / \phi^{-}}=-\frac{i e G_{F}^{S M}}{8 \sqrt{2} \pi^{2}} m_{l}\left\{\overline{u_{l}}(p-q)\left(P_{L}+2 P_{R}\right)(2 p \cdot \varepsilon) u_{l}(p)\right\} \frac{v^{2}}{2}\left(Y_{\Sigma}^{\dagger} M_{\Sigma}^{-1}\right)_{l i}\left(M_{\Sigma}^{-1} Y_{\Sigma}\right)_{i l} F_{1}\left(x_{i}\right) \\
& T_{\Sigma_{i}}^{W^{-} / W^{-}}=\frac{i e G_{F}^{S M}}{8 \sqrt{2} \pi^{2}} m_{l}\left\{\overline{u_{l}}(p-q)(2 p \cdot \varepsilon) u_{l}(p)\right\} \frac{v^{2}}{2}\left(Y_{\Sigma}^{\dagger} M_{\Sigma}^{-1}\right)_{l i}\left(M_{\Sigma}^{-1} Y_{\Sigma}\right)_{i l} F_{4}\left(x_{i}\right) \\
& T_{\Sigma_{i}}^{\phi^{-} / \phi^{-}}=\frac{i e G_{F}^{S M}}{8 \sqrt{2} \pi^{2}} m_{l}\left\{\overline{u_{l}}(p-q)(2 p \cdot \varepsilon) u_{l}(p)\right\} \frac{v^{2}}{2}\left(Y_{\Sigma}^{\dagger} M_{\Sigma}^{-1}\right)_{l i}\left(M_{\Sigma}^{-1} Y_{\Sigma}\right)_{i l} x_{i} F_{5}\left(x_{i}\right) \\
& T_{\Psi_{i}}^{Z}=\frac{i e G_{F}^{S M}}{8 \sqrt{2} \pi^{2}} m_{l}\left\{\overline{u_{l}}(p-q)(2 p \cdot \varepsilon) u_{l}(p)\right\} \frac{v^{2}}{2}\left(Y_{\Sigma}^{\dagger} M_{\Sigma}^{-1}\right)_{l i}\left(M_{\Sigma}^{-1} Y_{\Sigma}\right)_{i l} 2 G_{1}\left(y_{i}\right) \\
& T_{\Psi_{i}}^{H}=\frac{i e G_{F}^{S M}}{8 \sqrt{2} \pi^{2}} m_{l}\left\{\overline{u_{l}}(p-q)(2 p \cdot \varepsilon) u_{l}(p)\right\} \frac{v^{2}}{2}\left(Y_{\Sigma}^{\dagger} M_{\Sigma}^{-1}\right)_{l i}\left(M_{\Sigma}^{-1} Y_{\Sigma}\right)_{i l} y_{i} G_{2}\left(y_{i}\right) \\
& T_{\Psi_{i}}^{\eta}=\frac{i e G_{F}^{S M}}{8 \sqrt{2} \pi^{2}} m_{l}\left\{\overline{u_{l}}(p-q)(2 p \cdot \varepsilon) u_{l}(p)\right\} \frac{v^{2}}{2}\left(Y_{\Sigma}^{\dagger} M_{\Sigma}^{-1}\right)_{l i}\left(M_{\Sigma}^{-1} Y_{\Sigma}\right)_{i l} z_{i} G_{2}\left(z_{i}\right) \\
& T_{l_{i}^{\prime}}^{Z}=\frac{i e G_{F}^{S M}}{8 \sqrt{2} \pi^{2}} m_{l}\left\{\overline{u_{l}}(p-q)(2 p \cdot \varepsilon) u_{l}(p)\right\} \delta_{l_{i}^{\prime} l}\left[\left(G_{4}\left(y_{l_{i}^{\prime}}\right)-12 \cos ^{2} \theta_{W} G_{3}\left(y_{l_{i}^{\prime}}\right)\right.\right. \\
& \left.\left.+8 \cos ^{4} \theta_{W} G_{3}\left(y_{l_{i}^{\prime}}\right)\right)+\epsilon_{l l}\left(-4 G_{5}\left(y_{l_{i}^{\prime}}\right)+8 \cos ^{2} \theta_{W} G_{3}\left(y_{l_{i}^{\prime}}\right)\right)\right] \\
& T_{l_{i}^{\prime}}^{H}=0 \\
& T_{l_{i}^{\prime}}^{\eta}=0
\end{aligned}
$$


where $x_{\nu_{i}} \equiv \frac{m_{\nu_{i}}^{2}}{M_{W}^{2}}, x_{i} \equiv \frac{M_{\Sigma_{i}}^{2}}{M_{W}^{2}}, y_{l_{i}^{\prime}} \equiv \frac{m_{l_{i}^{\prime}}^{2}}{M_{Z}^{2}}, y_{i} \equiv \frac{M_{\Sigma_{i}}^{2}}{M_{Z}^{2}}, z_{i} \equiv \frac{M_{\Sigma_{i}}^{2}}{M_{H}^{2}}$ and $F_{i}(x)$ and $G_{i}(x)$ are the following functions:

$$
\begin{aligned}
& F_{1}(x)=J_{0}(x)-2 J_{1}(x)+J_{2}(x) \\
& F_{2}(x)=3 J_{0}(x)-8 J_{1}(x)+7 J_{2}(x)-2 J_{3}(x) \\
& F_{3}(x)=J_{1}(x)-J_{3}(x) \\
& F_{4}(x)=-9 J_{0}(x)+16 J_{1}(x)-5 J_{2}(x)-2 J_{3}(x) \\
& F_{5}(x)=-3 J_{1}(x)+4 J_{2}(x)-J_{3}(x) \\
& G_{1}(x)=7 I_{1}(x)-8 I_{2}(x)+I_{3}(x) \\
& G_{2}(x)=2 I_{0}(x)-3 I_{1}(x)+I_{3}(x) \\
& G_{3}(x)=I_{1}(x)-2 I_{2}(x)+I_{3}(x) \\
& G_{4}(x)=3 I_{1}(x)-8 I_{2}(x)+5 I_{3}(x) \\
& G_{5}(x)=3 I_{1}(x)-4 I_{2}(x)+I_{3}(x)
\end{aligned}
$$

where 7

$$
J_{n}(x)=\int_{0}^{1} \mathrm{~d} \alpha \frac{\alpha^{n}}{1-\alpha(1-x)} \quad I_{n}(x)=\int_{0}^{1} \mathrm{~d} \alpha \frac{\alpha^{n}}{x+\alpha(1-x)} .
$$

In the limit in which $x_{\nu_{i}} \rightarrow 0$ and $y_{l} \rightarrow 0$, taking the linear terms in $x_{\nu_{i}}$, we have:

$$
\begin{array}{lll}
F_{1}\left(x_{\nu_{i}}\right) \rightarrow \frac{1}{2}-\frac{1}{2} x_{\nu_{i}} & F_{2}\left(x_{\nu_{i}}\right) \rightarrow \frac{7}{6}-\frac{5}{6} x_{\nu_{i}} & F_{3}\left(x_{\nu_{i}}\right) \rightarrow \frac{5}{6}+\frac{10}{3} x_{\nu_{i}} \\
G_{3}\left(y_{l}\right) \rightarrow \frac{1}{3} & G_{4}\left(y_{l}\right) \rightarrow \frac{2}{3} & G_{5}\left(y_{l}\right) \rightarrow \frac{4}{3} .
\end{array}
$$

In this limit, grouping the various amplitudes according to the fermion circulating in the loop and summing over $i$, we get the results displayed in the text, Eqs. (14)-(17).

\section{References}

[1] A. Czarnecki and W. J. Marciano, Phys. Rev. D 64 (2001) 013014 arXiv:hep-ph/0102122]; K. Melnikov and A. Vainshtein, "Theory of the muon anomalous magnetic moment," Berlin, Germany: Springer (2006) and references therein.

[2] D. Stockinger, J. Phys. G 34 (2007) R45 arXiv:hep-ph/0609168 and references therein.

[3] M. Passera, Nucl. Phys. Proc. Suppl. 169 (2007) 213 arXiv:hep-ph/0702027.

\footnotetext{
${ }^{7}$ The terms involving $x_{l}=m_{l}^{2} / M_{W}^{2}$ and $y_{l}=m_{l}^{2} / M_{Z}^{2}$ in the denominators of $J_{n}$ and $I_{n}$ have been neglected.
} 
[4] J. S. Schwinger, Phys. Rev. 73 (1948) 416; C.M. Sommerfield, Phys. Rev. 107 (1957) 328 and Ann. Phys. (N.Y.) 5 (1958) 26; A. Petermann, Helv. Phys. Acta 30 (1957) 407 and Nucl. Phys. 5 (1958) 677; M. Passera, Phys. Rev. D 75 (2007) 013002.

[5] P.J. Mohr and B.N. Taylor, Rev. Mod. Phys. 77 (2005) 1.

[6] B. C. Odom, D. Hanneke, B. D’Urso and G. Gabrielse, Phys. Rev. Lett. 97 (2006) 030801 [Erratum-ibid. 99 (2007) 039902].

[7] G. W. Bennett et al. [Muon g-2 Collaboration], Phys. Rev. Lett. 92 (2004) 161802 |arXiv:hep-ex/0401008]; Phys. Rev. D 73 (2006) 072003 [arXiv:hep-ex/0602035].

[8] K. Hagiwara, A.D. Martin, D. Nomura and T. Teubner, hep-ph/0611102.

[9] M. Davier, S. Eidelman, A. Höcker and Z. Zhang, Eur. Phys. J. C 31 (2003) 503.

[10] F. Jegerlehner, Nucl. Phys. Proc. Suppl. 162 (2006) 22; J.F. de Trocóniz and F.J. Ynduráin, Phys. Rev. D 71 (2005) 073008.

[11] J. Abdallah et al. [DELPHI Collaboration], Eur. Phys. J. C 35 (2004) 159 arXiv:hep-ex/0406010.

[12] P. Minkowski, Phys. Lett. B 67421 (1977); M. Gell-Mann, P. Ramond and R. Slansky, in Supergravity, edited by P. van Nieuwenhuizen and D. Freedman, (North-Holland, 1979), p. 315; T. Yanagida, in Proceedings of the Workshop on the Unified Theory and the Baryon Number in the Universe, edited by O. Sawada and A. Sugamoto (KEK Report No. 79-18, Tsukuba, 1979), p. 95; R.N. Mohapatra and G. Senjanović, Phys. Rev. Lett. 44 (1980) 912.

[13] R. Foot, H. Lew, X. G. He and G. C. Joshi, Z. Phys. C 44 (1989) 441.

[14] A. Abada, C. Biggio, F. Bonnet, M. B. Gavela and T. Hambye, Phys. Rev. D 78 (2008) 033007 arXiv:0803.0481 [hep-ph]].

[15] S. Heinemeyer, W. Hollik and G. Weiglein, Phys. Rept. 425 (2006) 265 arXiv:hep-ph/0412214.

[16] R. Jackiw and S. Weinberg, Phys. Rev. D 5 (1972) 2396; I. Bars and M. Yoshimura, Phys. Rev. D 6 (1972) 374; G. Altarelli, N. Cabibbo and L. Maiani, Phys. Lett. B 40 (1972) 415; W. A. Bardeen, R. Gastmans and B. Lautrup, Nucl. Phys. B 46 (1972) 319; K. Fujikawa, B. W. Lee and A. I. Sanda, Phys. Rev. D 6 (1972) 2923.

[17] W. M. Yao et al. [Particle Data Group], J. Phys. G 33 (2006) 1 (and 2007 partial update for edition 2008). 
[18] A. Abada, C. Biggio, F. Bonnet, M. B. Gavela and T. Hambye, JHEP 12 (2007) 061 arXiv:0707.4058 [hep-ph]].

[19] J. Kersten and A. Y. Smirnov, Phys. Rev. D 76 (2007) 073005 arXiv:0705.3221 [hep-ph]].

[20] S. Antusch, C. Biggio, E. Fernandez-Martinez, M. B. Gavela and J. Lopez-Pavon, JHEP 0610 (2006) 084 arXiv:hep-ph/0607020].

[21] W. Chao, arXiv:0806.0889 [hep-ph]. 